\title{
Study on Angelica and its different extracts by Fourier transform infrared spectroscopy and two-dimensional correlation IR spectroscopy
}

\author{
Hong-xia Liu ${ }^{a}$, Su-qin Sun ${ }^{\text {a,* }}$, Guang-hua Lv ${ }^{b}$, Kelvin K.C. Chan ${ }^{b}$ \\ ${ }^{a}$ Department of Chemistry, Tsinghua University, Beijing 100084, China \\ ${ }^{\mathrm{b}}$ Research and Development Division, School of Chinese Medicine, Hong Kong Baptist University, Hong Kong, China
}

Received 17 April 2005; received in revised form 11 July 2005; accepted 12 July 2005

\begin{abstract}
In order to develop a rapid and effective analysis method for studying integrally the main constituents in the medicinal materials and their extracts, discriminating the extracts from different extraction process, comparing the categories of chemical constituents in the different extracts and monitoring the qualities of medicinal materials, we applied Fourier transform infrared spectroscopy (FT-IR) associated with second derivative infrared spectroscopy and two-dimensional correlation infrared spectroscopy (2D-IR) to study the main constituents in traditional Chinese medicine Angelica and its different extracts (extracted by petroleum ether, ethanol and water in turn). The findings indicated that FT-IR spectrum can provide many holistic variation rules of chemical constituents. Use of the macroscopical fingerprint characters of FT-IR and 2D-IR spectrum can not only identify the main chemical constituents in medicinal materials and their different extracts, but also compare the components differences among the similar samples. This analytical method is highly rapid, effective, visual and accurate for pharmaceutical research.
\end{abstract}

(C) 2005 Elsevier B.V. All rights reserved.

Keywords: Angelica sinensis (Oliv.) Diels; Fourier transform infrared spectroscopy (FT-IR); Second derivative infrared spectroscopy; Two-dimensional infrared correlation spectroscopy (2D-IR); Composition analysis; Macroscopic fingerprint

\section{Introduction}

In recent years, many extract products of medicinal materials come into the medicinal market, those concentrated extract products can not only enhance the curative effect, but also provide convenience for the medicine package, transport and carrying. So, the use of extract products of medicinal materials becomes more and more extensive. However, some counterfeits or products with poor qualities emerge in the medicinal market along with the popularity of extract products of medicinal materials. At present, the main method for identifying the qualities of medicinal materials and extract products is to use chromatography examining the content of certain chemical constituent in the tested sample. It is well known that the medicinal materials comprise hundreds of components, and produce their curative effects through

\footnotetext{
* Corresponding author. Tel.: +86 106278 7661; fax: +86 1062770327

E-mail address: sunsq@ chem.tsinghua.edu.cn (S.-q. Sun).
}

mutual effects of many ingredients, so the limited numbers of specific components cannot availably reflect the real qualities of the herbal medicines. Therefore, we starve for a quick and effective analysis method to entirely monitor and reflect the whole constituents of the medicinal materials and their corresponding extract products.

FT-IR is one of the most widely used methods to identify the chemical constituents and elucidate the compounds structures, and has been used as a requisite method to identify medicines in Pharmacopoeia of many countries. Owing to the fingerprint characters and extensive applicability to the samples, FT-IR has played an important role in pharmaceutical analysis in recent years [1-3]. The modernization of traditional Chinese medicine requires a quick and effective analysis method used for the complicated mixture system. Along with the development of FT-IR spectroscopic technique, as well as combined with the computer science, FT-IR has done work in the traditional Chinese medicine research [4,5]. Especially, Isao Noda established two-dimensional cor- 
relation infrared spectroscopy (2D-IR) in 1986 and then put forward a generalized 2D-IR in 1993 [6], which made it possible to apply FT-IR to analyze the complicated system. As long as the samples are different in components or contents, the differences will be embodied in their FT-IR spectra. The three steps of spectral analysis, FT-IR, second derivative infrared spectroscopy and then 2D-IR, can not only identify the major components in medicinal materials and their extracts, but also find the tiny differences among the similar samples.

The root of Angelica sinensis (Oliv.) Diels (Umbellifeae), known as Danggui in China, is a famous traditional Chinese medicine in common use [7]. It was reported that the active constituents in Angelica included essential oil and watersoluble substances [8]. Modern pharmacological research indicated that it could be used to treat anaemia, female disease, apoplexy, hypertension, coronary heart disease, thromboangiitis obliterans, superficial thrombosed phlebitis, etc. [9]. It was also used in cosmetic trade [10]. Therefore, many researchers at home and abroad are interested in studying Angelica and now some new cognition on the effective constituents and functions about Angelica have been acquired. In this article, we report the main components and the holistic variation rules of chemical constituents in Angelica and its extracts by applying FT-IR, second derivative infrared spectroscopy and 2D-IR. At last, we want to develop a rapid, accurate and feasible analysis method to integrally reflect the inherent qualities of medicinal materials and their corresponding extract products.

\section{Experiment}

\subsection{Apparatus and reagents}

Spectrometer: Spectrum GX FTIR system (Perkin-Elmer, USA), equipped with a DTGS detector. Scan range: from 400 to $4000 \mathrm{~cm}^{-1}$ with a resolution of $4 \mathrm{~cm}^{-1}$. Spectra were obtained from the accumulation of a total of 32 scans. Temperature controller: portable programmable temperature controller (Model 50-886, Love Control). Range: room temperature to $120^{\circ} \mathrm{C}$.

Reagents: petroleum ether (bp $60-90^{\circ} \mathrm{C}$ ), $95 \%$ ethanol were all analytical reagents (A.R.).

\subsection{Samples}

The roots of Angelica sinensis (Oliv.) Diels (whole root, first grade) were provided and identified by Ms. W.M. Tam (Hong Kong Institute of Oversea Chinese Medicinal Material, Hong Kong, China) in March 2001. The samples were harvested from Minxian, Gansu province, China in 2000.

\subsection{Procedure}

The dried roots of Angelica sinensis (Oliv.) Diels (200 g) was pulverized into coarse powder. One-eighth of the coarse powder was further ground and passed through a 200-mesh sieve (sample a). Seven-eighth of coarse powder was orderly extracted by petroleum ether (bp $60-90{ }^{\circ} \mathrm{C}$ ), $95 \%$ ethanol and water under reflux for three times, respectively. The petroleum ether extract was condensed under vacuum as yellow oil (sample b, $3.50 \mathrm{~g}$ ). The alcoholic extract and aqueous extract were condensed under vacuum and dried as cream (sample c, $19.25 \mathrm{~g}$ and sample d, $85.75 \mathrm{~g}$ ), respectively. The material residue was dried, ground and then passed through a 200-mesh sieve (sample e, $59.50 \mathrm{~g}$ ). All extracts were frozen drying except the petroleum ether extract. After that each of the extracts was coated on $\mathrm{KBr}$ blocky crystals, then ground the $\mathrm{KBr}$ crystals and pressed into a tablet, and then the infrared spectra of all samples were collected.

For collecting the dynamic FT-IR spectra, the samples tablets of aqueous extract and alcoholic extract were respectively put into the pool of the temperature controller to record the IR spectra in situ. A pre-established program was used to control the whole process of temperature increasing. The IR spectra were collected from room temperature to $120^{\circ} \mathrm{C}$ at intervals of $10^{\circ} \mathrm{C}$.

All the second derivative IR spectra were obtained after 13-point smoothing of the original IR spectra at room temperature.

2D-IR spectra were obtained by treatment of the series of dynamic spectra with 2D-IR correlation analysis software developed in our lab.

\section{Results and discussion}

Table 1 gives the lixiviation ratio of different extracts. It shows that the proportion of aqueous extract is the most $(49 \%, w / w)$. The FT-IR spectra of Angelica raw material, its different extracts and the extracted residue all have different macroscopic features (Fig. 1). From the spectra we can see clearly that although they show substantial overlap of each absorption spectrum of various components, each band represents an overall overlap of some characteristic absorption peaks of functional groups in the samples. In Fig. 1, the FTIR spectra of Angelica materials (a), alcoholic extract (c) and aqueous extract (d) are rather similar, which obviously differ from the spectra of petroleum ether extract (b) and extracted residue (e). All the FT-IR spectra of the tested samples were respectively analyzed in what follows.

It should be pointed that the peak at $\sim 1635 \mathrm{~cm}^{-1}$ may be interfered by the presence of moisture, so the peak nearby this position does not been assigned.

Table 1

The lixiviation ratio of different extracts

\begin{tabular}{lc}
\hline Extracts & Lixiviation ratio $(\%, \mathrm{w} / \mathrm{w})$ \\
\hline Petroleum ether & 2 \\
Ethanol & 11 \\
Water & 49 \\
Residue & 34 \\
\hline
\end{tabular}




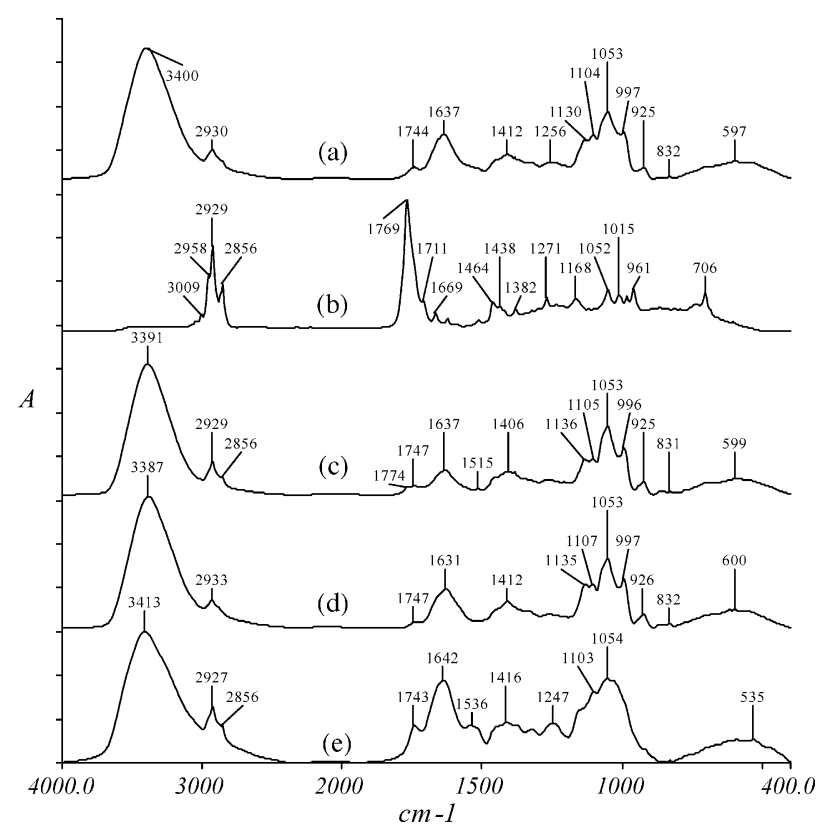

Fig. 1. FT-IR spectra of Angelica and its different extracts: (a) Angelica; (b) petroleum ether extract; (c) alcoholic extract; (d) aqueous extract; (e) extracted residue.

\subsection{IR spectra analysis of Angelica materials}

Although the interferences of plant fibers inherent in medicinal materials, we can still see that the IR spectrum of Angelica shows lots of structural information of major constituents. As shown in Fig. 1a, except the strongest peak at $3400 \mathrm{~cm}^{-1}$ assigned to the $\mathrm{O}-\mathrm{H}$ stretching vibration, the stronger peaks appear in the range of $1130-997 \mathrm{~cm}^{-1}$ mainly attributed to the stretching vibration of $\mathrm{C}-\mathrm{O}$. Those characters of peaks intensities and positions at 2930, 1130, 1104, 997,925 and $832 \mathrm{~cm}^{-1}$ in the IR spectrum display the characteristic absorptions of polysaccharides, especially the peak at $832 \mathrm{~cm}^{-1}$ belongs the characteristic peak of $\alpha$-glucose [11]. Angelica polysaccharides are one of the main effective constituents which have the obvious functions on enhancing body immunity, antitumor and resisting radiation damage [11]. In addition, the peak at $1744 \mathrm{~cm}^{-1}$ assigned to the $\mathrm{C}=\mathrm{O}$ stretching vibration means that some carbonyl compounds existed in Angelica. So, depending on the fingerprint characters of the peaks positions, shapes and intensities, the fundamental components indwell in the materials can been seen clearly.

\subsection{IR spectra analysis of petroleum ether extract}

The IR spectrum of petroleum ether extract (Fig. 1b) is distinctly different from those of alcoholic extract and aqueous extract (Fig. 1c and d). The obvious differences are the hydroxyl peak at $\sim 3400 \mathrm{~cm}^{-1}$ cannot be seen in it and the peak situated at $1769 \mathrm{~cm}^{-1}$ assigned to the absorption of carbonyl becomes the strongest one, as well as the peaks at 2958, 2929 and $2856 \mathrm{~cm}^{-1}$ get stronger, which belong to the $\mathrm{C}-\mathrm{H}$ stretching vibration of methyl and methylene; meanwhile, the peak intensity at $1052 \mathrm{~cm}^{-1}$ is relatively weak. Those characters are the typical absorption of lipophilic components [12]. In addition, some weaker peaks of carbonyl at 1711 and $1669 \mathrm{~cm}^{-1}$ can also be seen in Fig. 1b. The analysis results of GC-MS [13] indicated that the major constituents in Angelica lipophilic components are the compounds of ligustilides $(45 \%, w / w)$ which have the $\gamma$-lactone skeleton in molecular structure. As we known, the size of lactone ring can be inferred by the position of the carbonyl [14], so the strongest peak of carbonyl $\left(v_{\mathrm{C}=\mathrm{O}}\right)$ at $1769 \mathrm{~cm}^{-1}$ in Fig. $1 \mathrm{~b}$ means that many $\gamma$-lactone compounds exist in the petroleum ether extract, and the findings are consistent with the results of the literature report.

In sum, these notable peaks in Fig. 1b illuminate that there are lots of lipophilic components in the petroleum ether extract, such as essential oil, micromolecule terpenoids, greases, fatty acids and their esters which contain mostly the functional groups of carbonyl and alkyl.

\subsection{Analysis of IR spectra, second derivative spectra and $2 D$-IR spectra of the alcoholic extract and the aqueous extract}

Comparison of the two IR spectra of the alcoholic extract and the aqueous extract (Fig. 1c and d) reveals that the peaks positions and shapes of the main specific bands in the spectra are quite similar to each other. Both of them display the classific absorptions of polysaccharides at the frequencies of $\sim 3390, \sim 1135, \sim 1105, \sim 1053, \sim 996, \sim 925$ and $\sim 831 \mathrm{~cm}^{-1}$, just like the peaks in the Angelica medicinal materials mentioned above. The main differences between the two extracts are that there are two carbonyl peaks at $1774 \mathrm{~cm}^{-1}, 1747 \mathrm{~cm}^{-1}$ and one relatively obvious peak of $-\mathrm{CH}_{2}-$ at $2856 \mathrm{~cm}^{-1}$ in the spectrum of alcoholic extract (Fig. 1c), while there is only one carbonyl peak at $1747 \mathrm{~cm}^{-1}$ in the spectrum of the aqueous extract (Fig. 1d). These data indicate that the types of carbonyl compounds in the alcoholic extract are more than those in the aqueous extract owing to the different polarities of extraction solvents. However, their tiny differences make it difficult to identify unambiguously them by utilizing the conventional FT-IR technique only.

Generally, the second derivative infrared spectrum can obviously enhance the spectral resolution and amplify tiny differences in IR spectrum. The second derivative spectra of the alcoholic extract and the aqueous extract are shown in Fig. 2. Many differences invisible in FT-IR spectra become clearer; especially in the range from 1300 to $1800 \mathrm{~cm}^{-1}$, the numbers of absorption peaks in the alcoholic extract are more than those in the aqueous extract. For example, the obvious peaks at $1558,1517,1467 \mathrm{~cm}^{-1}$ in the alcoholic extract cannot be found in the aqueous extract, as well as the peaks intensities and positions are different between the two extracts in the range from 1380 to $1450 \mathrm{~cm}^{-1}$. In addition, the carbonyl differences are enlarged in the secondary derivative spectra of the alcoholic extract (1774 and $\left.1747 \mathrm{~cm}^{-1}\right)$ and 


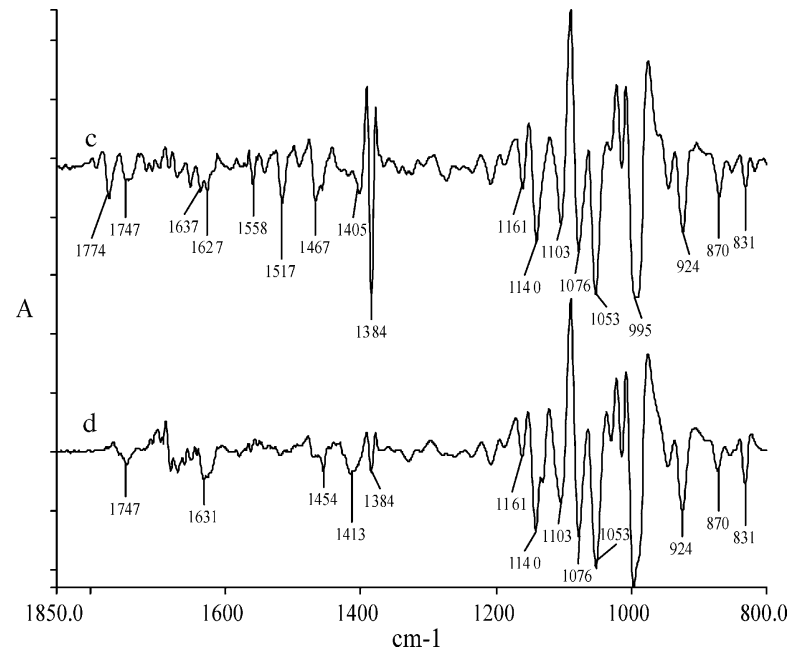

Fig. 2. Second derivative spectra of the alcoholic extract (c) and the aqueous extract (d).

the aqueous extract $\left(1747 \mathrm{~cm}^{-1}\right)$, and the peak of $\alpha$-glucose at $831 \mathrm{~cm}^{-1}$ becomes legible compared with the FT-IR spectra. Hence, these two extracts can be identified easily by those enlarged fingerprint characters in the secondary derivative IR spectra.

It has been shown that 2D-IR spectrum can enhance the spectral resolution and obtain much new information which cannot be acquired from IR spectrum and second derivative spectrum [15]. Therefore, it can provide visual and legible spectra for discriminating the similar complicated samples.

The differences of chemical constituents between the alcoholic extract and the aqueous extract can be described further through the 2D-IR synchronous spectra (Figs. 3 and 4). The left graph is plane figure, and the right graph is stereo-fish-net figure. The correlation peaks in synchronous spectrum represent a consistency or commonality of structural changes of the related IR vibrations with some perturbations such as temperature. In synchronous spectrum, the auto-peaks on the diagonal line show the self-correlativity and susceptibility of some normal vibration of functional group with the increasing temperature, and the cross peaks located at the off-diagonal position reveal the relativity of intensity variations of a pair of group vibrations corresponding their frequencies. The closer the correlativity is, the stronger the intensity of auto-peak will be [16]. We can see clearly some differences between the alcoholic extract and the aqueous extract in the 2D-IR spectra. For example, there is only one distinct auto-peak at $1450 \mathrm{~cm}^{-1}$ in synchronous spectrum of the alcoholic extract within $1200-1500 \mathrm{~cm}^{-1}$ (Fig. 3), while in the aqueous extract there are not only two autopeaks situated at 1225 and $1450 \mathrm{~cm}^{-1}$, but also their intensities are stronger than that in the alcoholic extract, so they have stronger self-correlativity in the increasing temperature process.

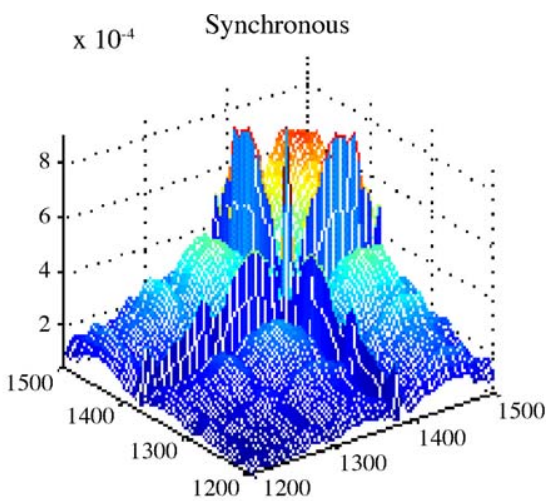

(c)

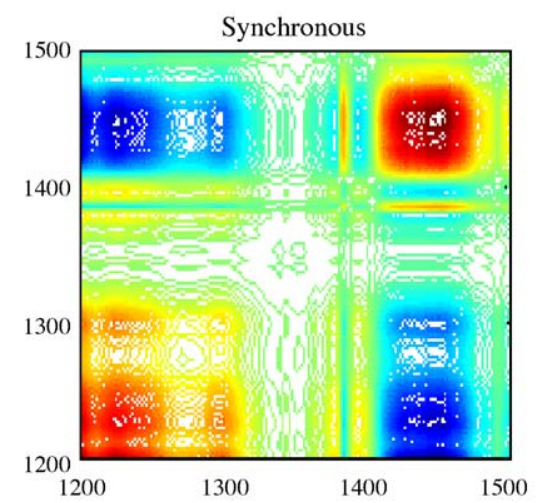

(d)

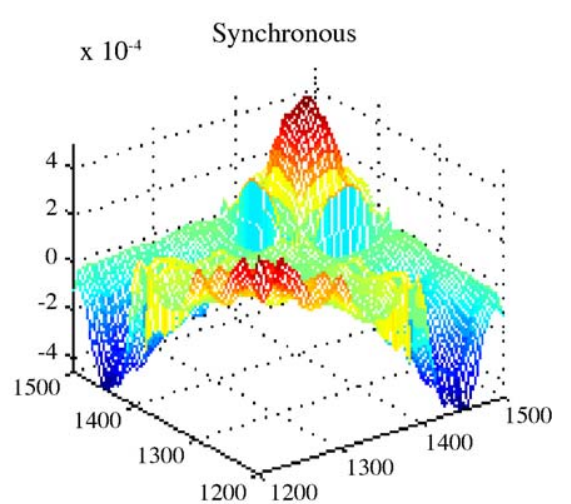

Fig. 3. 2D-IR spectra of the alcoholic extract (c) and the aqueous extract (d) (range: $1200-1500 \mathrm{~cm}^{-1}$ ). 

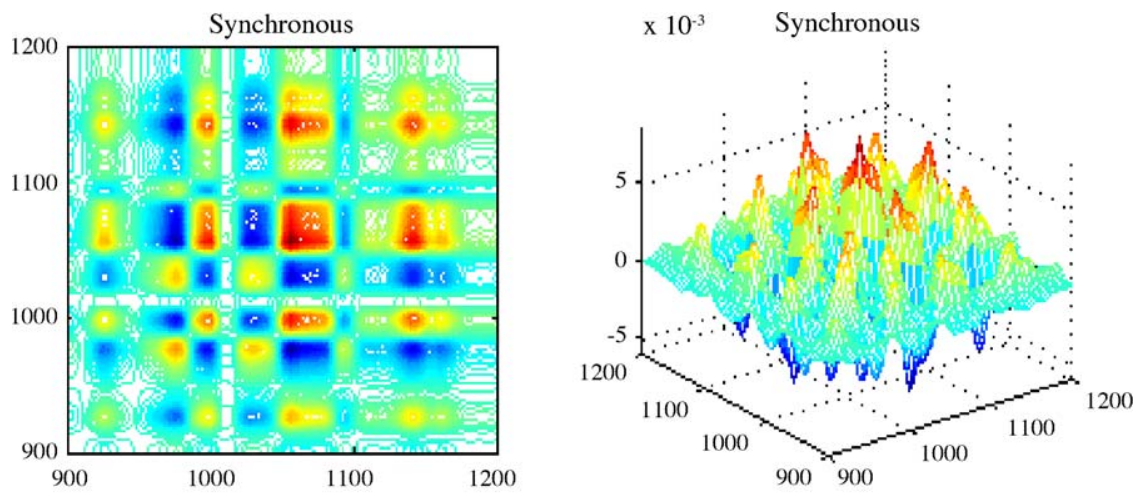

(c)
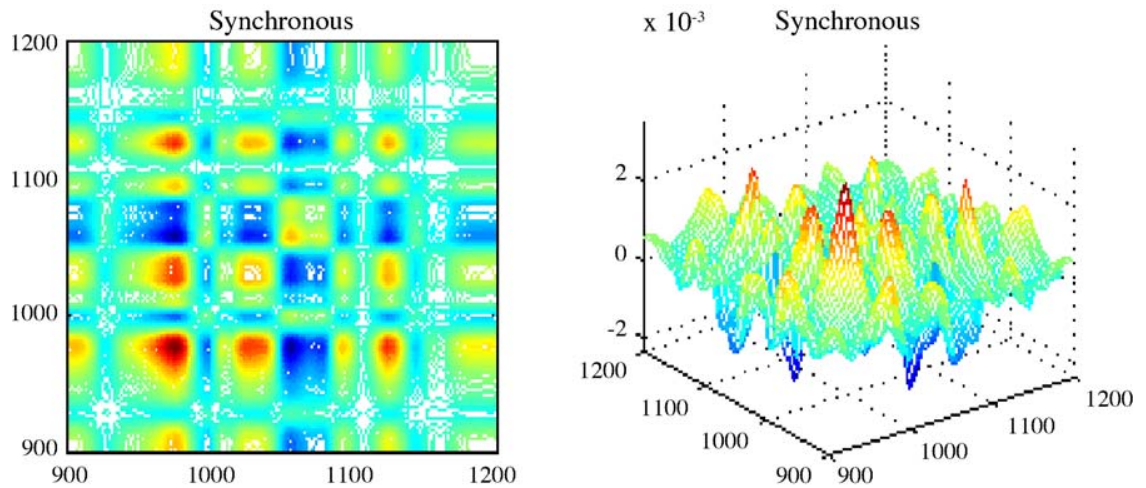

(d)

Fig. 4. 2D-IR spectra of the alcoholic extract (c) and the aqueous extract (d) (range: $900-1200 \mathrm{~cm}^{-1}$ ).

The alcoholic extract and the aqueous extract have very similar FT-IR and second derivative spectra in the range of $900-1200 \mathrm{~cm}^{-1}$, which mainly reflects the absorption of $\mathrm{C}-\mathrm{O}$ stretching vibration and the skeleton stretching vibration of glucides. However, the 2D-IR spectra at the same range (Fig. 4) indicate that the polysaccharides in these two extracts are very different. Six auto-peaks at 923, 996, 1004, 1023,1053 and $1145 \mathrm{~cm}^{-1}$ can be seen in the spectrum of alcoholic extract, in which the strongest peak situates at $1053 \mathrm{~cm}^{-1}$, while in the spectrum of aqueous extract there are only four auto-peaks appear at 996, 1042, 1053 and $1130 \mathrm{~cm}^{-1}$, and in which the strongest peak is at $996 \mathrm{~cm}^{-1}$. The spectral differences are the objective embodiment of the differences of chemical constituents in the samples, so we know the polysaccharides in the alcoholic extract are different from those in the aqueous extract.

\subsection{Comparison of IR spectra of the extracted residue and Angelica materials}

Lignin, cellulose and hemicellulose are the main compositions of botanical skeleton. The compositions in plant cell wall consist of cellulose, pectin, hemicellulose, and glycoprotein [17]. The absorption peaks of plant fiber polysaccharides, glycoprotein and vegetable protein inherent in the extracted residue are visible after the main chemical constituents in
Angelica were extracted out systematically by multi-solvents. As shown in Fig. 5e, in the extracted residue the strongest peak of $v_{\mathrm{O}-\mathrm{H}}$ at $3413 \mathrm{~cm}^{-1}$ and the stronger peak of $v_{\mathrm{C}-\mathrm{O}}$ at $1054 \mathrm{~cm}^{-1}$ are the typical absorption peaks of cellulose or hemicellulose, which are obviously different from the peaks of polysaccharides in the Angelica materials (Fig. 5a). While the stronger peak at $1642 \mathrm{~cm}^{-1}$ and the new peak at $1536 \mathrm{~cm}^{-1}$ assigned respectively to amide I band $\left(v_{\mathrm{C}=\mathrm{O}}\right)$ and amide II band $\left(\delta_{\mathrm{NH}}+v_{\mathrm{CN}}\right)$ of glycoprotein [14] appear very

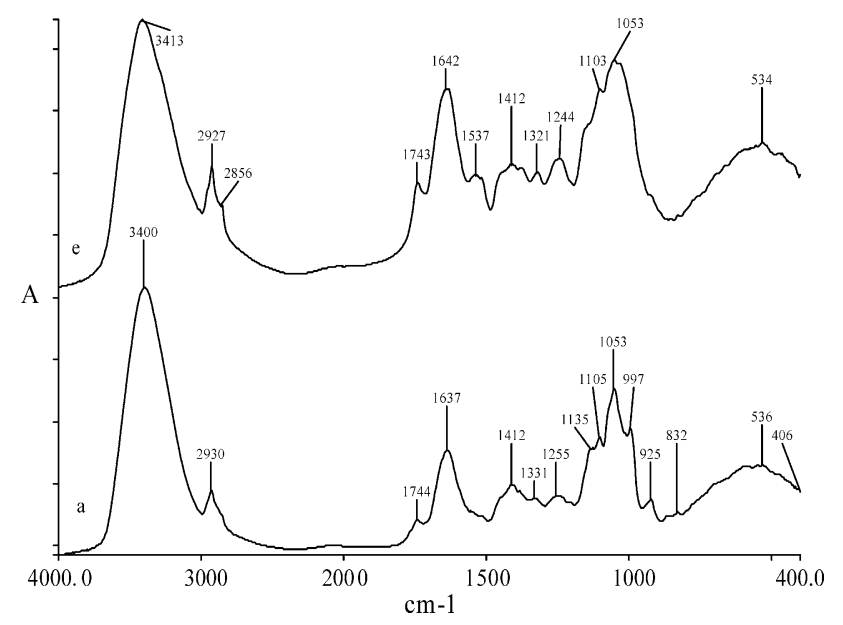

Fig. 5. FT-IR spectra of the extracted residue (e) and Angelica materials (a). 
clear in the extracted residue, which can hardly be seen in the Angelica materials. In addition, some carbonyl compounds still remain in the extracted residue, which are shown as the band at $1743 \mathrm{~cm}^{-1}$. So, it tells us that the compositions in the botanic skeleton, such as lignin, cellulose, hemicellulose and glycoprotein inherent in plants, are high-visible in the FT-IR spectrum of medicinal extracted residue. Hence, using these macroscopic characters of the two FT-IR spectra only can distinguish the extracted medicinal materials from the genuine ones quickly and easily. FT-IR spectra offer an effective means to appraise quickly the real qualities of the medicinal materials, e.g. traditional Chinese medicine, etc.

\section{Conclusion}

Based on the systematical analysis of Angelica, its different extracts and the extracted residue by using FT-IR, second derivative spectrum and 2D-IR, we know that those spectra can supply large numbers of macroscope structures information and holistic variation rules of chemical constituents in medicinal materials. This method reveals clearly the differences of categories of chemical constituents in Angelica and its different extracts. After the medicinal materials are extracted by solvents, the chemical compositions become relatively concentrated, so the FT-IR spectrum shows more characteristic features. Because of the commonality of the extraction methods, the chemical constituents in the extracts are relatively consistent, and then the FT-IR spectrum has higher repeatability and comparability. Spectral differences are the objective reflection of componential differences. By using the macroscope fingerprint characters of FT-IR spectrum we can judge accurately, fleetly and effectively the origin of different extracts, trace the constituents in the extracts, identify the medicinal materials true or false and even evaluate the qualities of medicinal materials. So, FT-IR spectrum reflecting objectively the panorama of chemical constituents in complex system is a most credible method to validate and identify the mix-substance systems such as traditional
Chinese medicine, herbal medicine, as well as their corresponding extract products.

\section{Acknowledgements}

This work is sponsored by the State Administration of Traditional Chinese Medicine of the People's Republic of China (2001ZDZX01) and grand support of Ministry of Science and Technology of the People's Republic of China (2002BA906A29-4).

\section{References}

[1] C.Y. Mu, J.Y. Shi, Shandong Med. Ind. 21 (2002) 23.

[2] M.A. Druy, Spectroscopy 19 (2004) 60.

[3] M.R. Sohrabi, M. Davallo, F. Tadayyon, F. Nabipoor, A. Khamneifar, Asian J. Chem. 17 (2005) 541.

[4] L. Zuo, S.Q. Sun, Q. Zhou, J.X. Tao, I. Noda, J. Pharm. Biomed. Anal. 30 (2003) 1491.

[5] S.Q. Sun, Q. Zhou, J. Liu, H. Huang, Spectrosc. Spectral Anal. 24 (2004) 427.

[6] I. Noda, Appl. Spectrosc. 47 (1993) 1329.

[7] Committee of National Pharmacopoeia, Pharmacopoeia of People's Republic of China, Chemical Industry Press, Beijing, 2000, p. 102.

[8] C.Y. Liu, L.H. Tang, Z.L. Gu, Chinese Wild Plant Resour. 16 (1997) 12.

[9] W.H. Huang, C.Q. Song, China J. Chin. Mater. Med. 26 (2001) 147.

[10] H.P. Ji, Gansu J. Trad. Chin. Med. 16 (2003) 37.

[11] R.J. Chen, G.Y. Xu, H.Y. Wang, H.Z. Xu, Y.M. Liu, Chem. Sin. 6 (2001) 372

[12] S.Q. Sun, Q. Zhou, Z. Qin, Two-dimensional Correlation Infrared Spectroscopy of Traditional Chinese Medicine, China Chemical Industry Press, Beijing, 2003, p. 26.

[13] Y. Dong, X.G. Wei, Q.X. Cui, G.L. Zhang, Shangdong J. Trad. Chin. Med. 23 (2004) 43.

[14] J.X. Xie, J.B. Chang, X.M. Wang, Infrared Spectrum, Science Press, Beijing, 2001, pp. 77, 212, 309.

[15] R. Hua, S.Q. Sun, Q. Zhou, I. Noda, B.Q. Wang, J. Pharm. Biomed. Anal. 33 (2003) 199

[16] I. Noda, J. Am. Chem. Soc. 111 (1989) 8116.

[17] R.X. Tan, J.C. Meng, D.F. Chen, Q.C. Jiao, Botanical Composition Analysis, Science Press, Beijing, 2002, p. 539. 